\title{
A TOUR OF THE LOCAL SUPERCLUSTER
}

R. Brent Tully and J. Richard Fisher

To complement our movie, we offer three steroscopic pictures of a region enclosing the Local Supercluster. A coordinate system is defined.

$X$ axis: in a line from the Virgo cluster to the Galaxy.

$\mathrm{Y}$ axis: orthogonal to $\mathrm{x}$ in the plane of the supercluster; the supercluster lies in the $x-y$ plane.

$\mathrm{Z}$ axis: perpendicular to the supercluster; the plane of the sky lies in the $y-z$ plane.

The distance scale is established assuming a uniform Hubble flow. The Virgo cluster is at the origin $(0,0,0)$. If $\mathrm{H}_{\mathrm{O}}=75 \mathrm{~km} \mathrm{~s}^{-1} \mathrm{Mpc}^{-1}$ is taken, then the Galaxy is at $(14.7,0,0)$. The boundaries of the volume displayed are then:

$$
\begin{aligned}
& -5<\mathrm{X}<14.7 \mathrm{Mpc} \\
& -14<\mathrm{Y}<17 \text { " } \\
& -10<\mathrm{Z}<10
\end{aligned}
$$

The pictures may be studied with a standard stero viewer or, with difficulty, by splitting the images with a card and viewing from a distance of about $15 \mathrm{~cm}$. The three perspectives are:

(a) a view essentially of the $y-z$ plane (in fact, there is a $13^{\circ}$ rotation about the $z$ axis),

(b) after a $30^{\circ}$ counterclockwise rotation about the $y$ axis from (a),

(c) after a $90^{\circ}$ counterclockwise rotation about the $y$ axis from (a), i.e. a view of the $x-y$ plane.

The viewer at vantage point (a) is edge-on to the disk of the Local Supercluster and the viewer at (c) is looking down from the pole. 

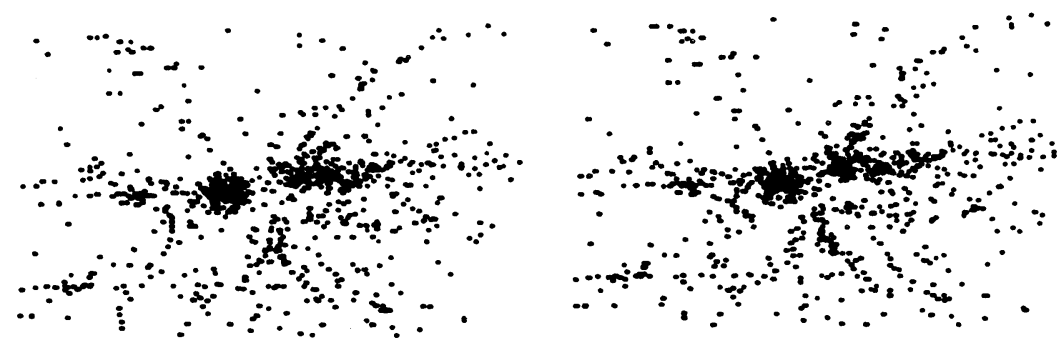

a
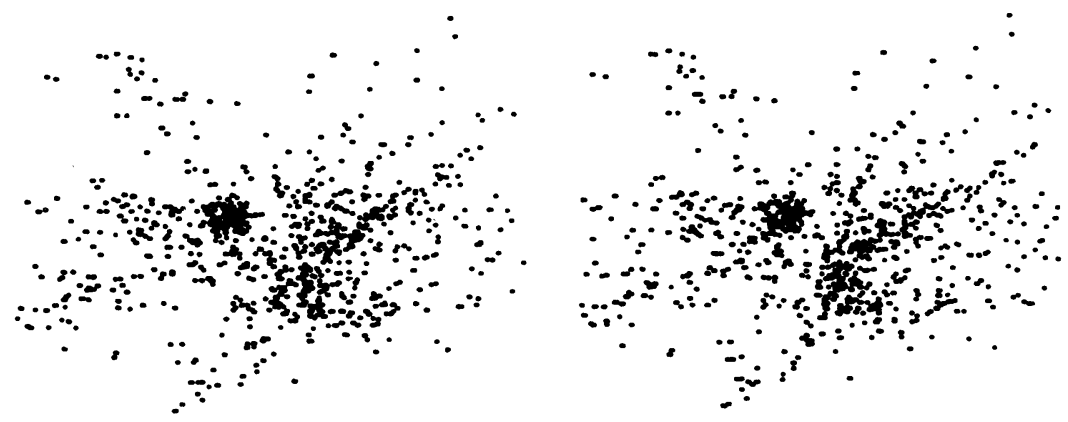

b
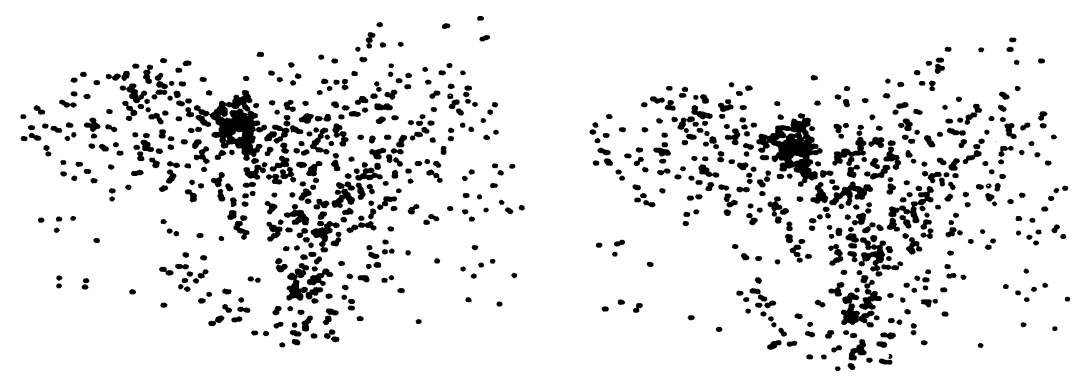

C 


\section{DISCUSSION}

de Vaucouleurs: Is the velocity range sufficient to cover the whole supercluster?

Tulzy: The Virgo cluster has a redshift of $1100 \mathrm{~km} \mathrm{~s}^{-1}$ while our outer boundary is at $3000 \mathrm{~km} \mathrm{~s}^{-1}$. The number density of galaxies beyond Virgo appears to fall off significantly faster than the fall off which would be expected from incompleteness in the sample. However, we could get into semantic difficulties about just what we mean by the term supercluster.

Abe Z2: In seeing this extremely interesting film, I had the impression, perhaps incorrect, that many of the groups and clusters of points seemed elongated towards us (i.e., the Galaxy). Can this not be an effect of velocity dispersion within the groups?

TuZzy: There are quite a number of elongated features but I am not aware of a tendency for them to point toward us. Of course, there is the very prominent region in Ursa Major - Canus Venatici which extends away from us, but in this region we know from non-redshift distance indicators that the low redshift objects are nearer and the high redshift objects are farther away. 